\title{
Effect of High Temperature Impact on The Formation of Adiabatic Shear Bands
}

\author{
Ali Eliasu ${ }^{1 *}$ and Solomon Boakye-Yiadom ${ }^{1}$ \\ ${ }^{1}$ York University, Department of Mechanical Engineering, Toronto, Canada. \\ * Corresponding author: alieliasu0101@gmail.com
}

It has been shown that a large percentage of the work done during plastic deformation of metals at high strain rates is converted to heat [1-2]. Investigations on the heat generated due to plastic deformation reports that it leads to thermal softening (adiabatic heating) of the material under deformation within narrow zones of the material and results in the formation of Adiabatic Shear Bands (ASBs) [1-2]. According to these studies, during high strain rates of deformation, there is insufficient time for the dissipation of the heat generated from the material to the atmosphere or the surroundings [1]. This leads to a localized increase in the temperature of the material. The heat produced is retained in the regions or zones where it was created leading to stress collapse and hence strain localization in narrow bands. Even though this softening mechanism has been reported by many investigators, it is argued that the rise in temperature may not be significantly high to cause considerable effect on the mechanical and microstructural properties of the materials during dynamic deformation [3-5]. Hence, the concept of high temperature leading to strain localization and the formation of adiabatic shear bands has been extensively debated. Currently, the actual influence of the rise in temperature on the formation of adiabatic shear bands is still under investigation.

In this study, AISI 4340 steel specimens were initially heat treated at different temperatures followed by impact at room and high temperatures to determine the effect of deformation temperature on the formation of adiabatic shear bands. It was hypothesized that if the heat generated due to conversion of plastic work to heat during plastic deformation were significantly high and concentrated in narrow regions (regions of strain localization) within the steel specimens during impact, then the structure within the evolved shear bands should have evidence of this rise in temperature. After impact, the specimens were examined using optical and electron microscopy techniques. The steel specimens exhibited failure by the development of shear bands after impact at room and high temperatures. At room temperature impact, white-etching shear bands were formed and microhardness indentations on the regions within the shear bands indicated that they were significantly hard and brittle compared to the regions outside the shear bands. The evolved white-etching shear bands were characterized by refined grains, network of dislocation structures and carbide dissolution. Regions away from the shear bands had network of dislocation structures and heavily deformed grains.

On the other hand, well-defined black- and white-etching shear bands were observed in all the specimens impacted at $50 \mathrm{Kg} . \mathrm{m} / \mathrm{s}$ at high temperatures $\left(\geq 300{ }^{\circ} \mathrm{C}\right)$. The white-etching shear bands were surrounded by black-etching shear bands and microhardness indentations on the regions within the shear bands indicated that the white-etching shear bands were harder and more brittle than the black etching shear bands. Extensive microstructural analysis on the regions within the evolved shear bands in the steel specimens revealed that there were no "signatures" of a high rise in temperature within the evolved shear bands. In addition, there was no phase change within the shear bands. The regions within the evolved shear bands were made up of recrystallized grains and distinct reprecipitated carbides as shown in Figure 1. These carbides were distributed within the recrystallized grains with no observed dislocation networks or structures. This study demonstrates that if the heat generated due to conversion of plastic work to heat 
during plastic deformation were significantly high and concentrated in narrow regions within the material under high strain rates and large strains of deformation, then the structure within the shear bands in the steel specimens should have evidence of this rise in temperature. The observed structure within the shear bands at both room and high temperature impact did not lend credence to a significant rise in temperature. It is concluded that the presence of high strain energies within the evolved ASBs led to recrystallization of the structure within the shear bands when compared to regions outside the shear bands. In addition, the high hardness is attributed to the presence of re-precipitated carbides which were dissolved within the evolved shear bands.

\section{References:}

[1] B. Dodd in “Adiabatic Shear Localization”, ed. Bai, (Elsevier, London), p.193

[2] C. Z. Duan, Y. J. Cai and M. J. Wang, G. H. Li, Journal of Materials Science 48 (2009), p.897-902.

[3] S. Boakye-Yiadom, A. K. Khan and N. Bassim, Materials Science and Engineering A 615 (2014), p.373-394.

[4] S. Boakye-Yiadom, A. K. Khan and N. Bassim, Materials Science and Engineering A 605 (2014), p.270-285.

[5] S. Boakye-Yiadom and N. Bassim, Materials Science and Engineering A 711 (2017), p.182-194.

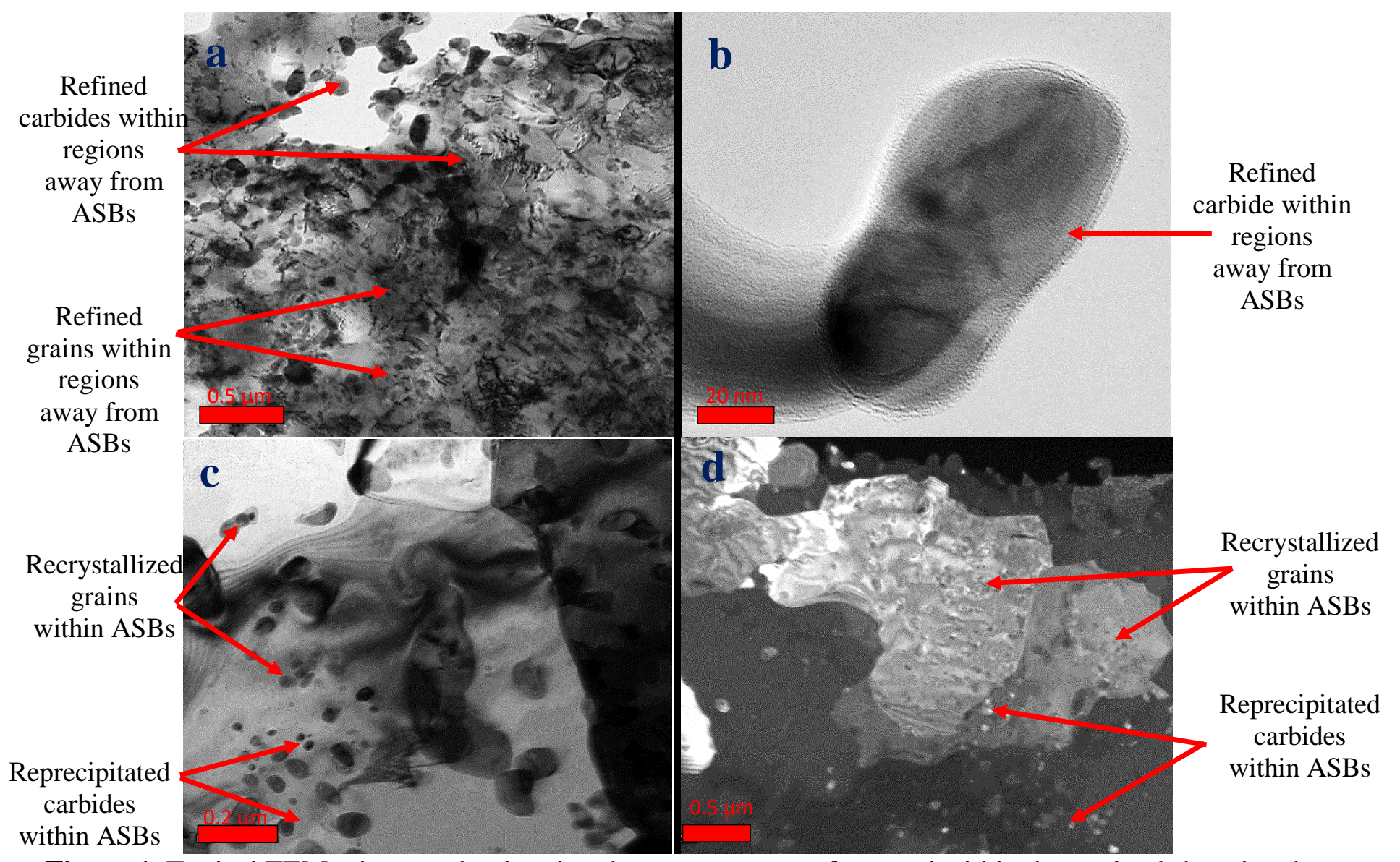

Figure 1. Typical TEM micrographs showing the structure away from and within the evolved shear bands in a steel specimen impacted at $50 \mathrm{kgm} / \mathrm{s}$ at $600^{\circ} \mathrm{C}$. (a), (b) Bright field images of regions away from evolved ASB. (c) Bright field (d) Dark field image of regions within evolved ASB. 